Purity Determination of Standard Analytical Reference Materials by Differential Scanning Calorimetry

Patrick B. Black and Dean Pidgeon 
For conversion of SImetric units to U.S./British customary units of measurement consult ASTM Standard E380, Metric Practice Guide, published by the American Society for Testing and Materials, 1916 Race St., Philadelphia, Pa. 19103. 


\section{Special Report 90-16}

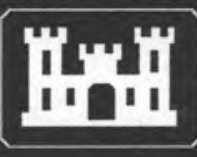

U.S. Army Corps of Engineers

Cold Regions Research \& Engineering Laboratory

\section{Purity Determination of Standard Analytical Reference Materials by Differential Scanning Calorimetry}

Patrick B. Black and Dean Pidgeon 


\section{PREFACE}

This report was prepared by Dr. Patrick B. Black, Soil Physicist, Applied Research Branch, Experimental Engineering Division, and Dean Pidgeon, Research Technician, Geochemical Sciences Branch, Research Division, U.S. Army Cold Regions Research and Engineering Laboratory.

Funding for this work was provided by the U.S. Army Toxic and Hazardous Materials Agency, Aberdeen Proving Ground, Maryland (R-90 Multi-Analytical Services), M. Stutz, Project Monitor.

The authors acknowledge the helpful suggestions of the technical reviewers, Dr. Samuel Colbeck and Daniel Leggett, as well as the technical assistance of Dr. Thomas Jenkins and Marianne Walsh.

The contents of this report are not to be used for advertising or promotional purposes. Citation of brand names does not constitute an official endorsement or approval of the use of such commercial products. 


\title{
Purity Determination of Standard Analytical Reference Materials by Differential Scanning Calorimetry
}

\author{
PATRICK B. BLACK AND DEAN PIDGEON
}

\section{INTRODUCTION}

Standard Analytical Reference Materials (SARMs) are a high-purity ( $>98 \mathrm{~mol} \%$ ) representative set of compounds used in the production of explosives (Table 1). Because the National Bureau of Standards has only a limited amount of these reference materials available for trace organic analysis, the United States Army Toxic and Hazardous Materials Agency (USATHAMA) has initiated a program for developing SARMs for use in its laboratories (USATHAMA 1987). This two-part program consists of procuring and producing the highpurity reference materials and then monitoring their purity level while in storage. This paper, which is concerned with the second part of the program, reports on the method of determining the purity of the seven SARMs noted in Table 1 that are suited to differential scanning calorimetry (DSC). The purity of the remaining SARMs are determined by other methods and will not be discussed further.

The USATHAMA Quality Assurance program manual (USATHAMA 1987) recommends a surveillance program that tests the purity of the SARMs at six-month intervals. For this project the criterion that is used for accepting the standard is that it does not have a purity level below $98 \mathrm{~mol} \%$. If the SARM fails to meet this criterion, the central repository is to be contacted and appropriate decisions made on its status.

The surveillance program for each SARM begins when the compound is first produced to the desired purity level, which is determined by successfully undergoing aggravated storage. An aggravated storage test consists of exposing the SARM to $70^{\circ} \mathrm{C}$ for two weeks in a loosely capped container followed by purity analysis by DSC.* The SARMs are then stored in an explosiveproof freezer, and their purity is monitored by DSC at

* Personal communication between T. Jenkins, CRREL, and M. Stutz, USATHAMA, 1989.
Table 1. SARMs analyzed.

\begin{tabular}{|c|c|c|c|}
\hline SARM & $\begin{array}{c}\text { Melting point } \\
\left({ }^{\circ} \mathrm{C}\right)\end{array}$ & $\begin{array}{l}\text { Heat of fusion } \\
\text { (cal/gm) }\end{array}$ & UsedDSC \\
\hline $1,3,5$ TNB & $122.5^{*}$ & & yes \\
\hline $2,4,6$ TNBA & & & yes \\
\hline $2,4 \mathrm{DNT}$ & $70^{+}$ & & yes \\
\hline $2,6 \mathrm{DNT}$ & & & yes \\
\hline Picric acid & $122.5^{\text {*** }}$ & $18.2^{\text {**** }}$ & yes: \\
\hline Tetryl & $\begin{array}{l}131^{*}, 129.5, t * * \\
129.45^{t+}\end{array}$ & $22.2^{\pi t}, 19.1^{* * *}$ & yes \\
\hline TNT & $\begin{array}{l}80.1^{\dagger}, 80.9,+1 \\
80.8^{* * *}\end{array}$ & $23.53^{t t}, 23.1^{* *}$ & yes \\
\hline Nitroglycerine & & & no \\
\hline RDX & & & no \\
\hline HMX & & & no \\
\hline Nitrocellulose & & & no \\
\hline
\end{tabular}

*Wentsel et al. (1979)

tJenkins et al. (1988)

**Meyer(1987)

ttGibbs and Popolato (1980)

six-month intervals. After two years the cycle is repeated by conducting another aggravated storage test (USATHAMA 1987).

This paper first describes the thermodynamic background and the underlying constraints that must be obeyed in order for molar purity to be determined by DSC techniques. Data on the thermophysical properties of the SARMs were collected according to the procedure presented by Pidgeon and Black (in prep.) and each SARM's purity was calculated. The accuracy of these calculated values were then evaluated and the final decision on the purity of each SARM made.

\section{THEORY OF MELTING OF HIGHLY PURE COMPOUNDS}

The melting of a pure compound is a first-order transition that occurs abruptly at a specific temperature 
$\theta_{\mathrm{o}}$ and involves a characteristic molar latent heat of fusion $\Delta L(\mathrm{cal} / \mathrm{mole})$. Minor amounts of impurities should lower the transition temperature to $\theta_{\mathrm{F}}$ as well as broaden the transition zone (Perkin-Elmer Corporation, n.d.). These changes are expressed by van't Hoff's equation (Plato and Glasgow 1969):

$$
\frac{\theta_{\mathrm{o}}-\theta_{\mathrm{F}}}{X_{2}}=R \frac{\theta_{\mathrm{o}}^{2}}{\Delta L}
$$

where $\theta_{F}=$ fusion temperature of the impure compound $\left({ }^{\circ} \mathrm{C}\right)$

$\theta_{\mathrm{o}}=$ melting point of the pure major component $\left({ }^{\circ} \mathrm{C}\right)$

$R=$ gas constant $\left(\mathrm{cal} \mathrm{mole}{ }^{-1}{ }^{\circ} \mathrm{C}^{-1}\right)$

$X_{2}=$ mole fraction of impurity $(\mathrm{mol} \mathrm{\%})$.

To correctly apply this form of the equation, several constraints must be obeyed (Hunter and Blaine 1984, Widmann and Sommerauer 1988):

- The mole fraction of impurity must be small $(<3 \%)$;

- The melted impurity must not form additional compounds with the major component as it completes total phase change; and

- The phase transition must be reversible.

The first constraint allows the implementation of the two mathematical simplifications that resulted in eq 1 : $\ln \left(1-X_{2}\right) \approx-X_{2}$; and $\theta_{F} \theta_{0} \approx \theta_{0}{ }^{2}$. Implicit in eq 1 as well is that $\Delta L$ is constant over the temperature range $\left(\theta_{0}-\theta_{F}\right)$. The second constraint assumes that the minor components are totally soluble in the liquid phase of the major component and totally insoluble in the solid phase of the major component. The final constraint is necessary to apply the equilibrium thermodynamics that leads to the van't Hoff's equation.

When a compound satisfies these three requirements, its purity can be determined by measuring, at most, its heat of fusion, its freezing point depression and its melting temperature. Differential scanning calorimetry offers an established method of concurrently measuring these three properties.

\section{EXPERIMENT}

A Perkin-Elmer DSC-4 differential scanning calorimeter was used to analyze the seven SARMs suited for DSC analysis (Table 1). The test method consisted of melting the test specimen at a carefully controlled temperature rate while recording the heat flow into the specimen as a function of time. The resulting endotherm was analyzed to determine the specimen's heat of fusion, melting temperature and freezing point depression, which
Table 2. Optimum sample parameters determined by Hunter and Blaine (1984).

\begin{tabular}{ll}
\hline Specimen size & $1.7 \mathrm{mg}$ \\
Heating rate & $0.5^{\circ} \mathrm{C} / \mathrm{min}$ \\
Data acquisition rate & 120 data points $/{ }^{\circ} \mathrm{C}$ \\
\hline
\end{tabular}

were then used in eq 1 to determine mole percent purity. The control of the DSC-4 and necessary data reduction were done using the procedure and program documented by Pidgeon and Black (in prep).

Throughout the several days during which the measurements were taken, an indium reference was regularly employed to calibrate the DSC-4 for temperature and thermal power. The measured calibration constants for the machine from each run were then used in determining the thermal properties of the test specimens measured following that calibration run.

ASTM standards proposed by Hunter and Blaine (1984) recommend optimum values of sample mass, heating rate and sampling rate for computer acquisition of impurity data. These conditions for an impurity $<2$ $\mathrm{mol} \%$ are presented in Table 2 . In addition to determining optimum parameters, Hunter and Blaine made two important observations. First, deviations from the optimum values result in underestimating the impurity level. Second, slight variations from these values may be tolerated, but the inaccuracies thus generated are cumulative. If deviations from optimum must occur, the "true" impurity will be larger than that calculated, and care must be taken to maintain the remaining parameters at their optimum if any one parameter must deviate.

Ten replicates of each compound were tested. Table 3 summarizes the results. A complete set of all the data and plots of each melting curve are presented in Pidgeon and Black (1989).

\section{DISCUSSION}

Based on these measurements alone, all compounds were determined to have a purity greater than $99 \mathrm{~mol} \%$. Therefore, these seven compounds meet the USA THAMA QA criterion for Standard Analytical Reference Materials.

The results in Table 3 show that the tests were not conducted at the optimum recommended settings of Table 2. This was due to the limitations of the data collection system used for the measurements. The system's limited memory required that a less-than-optimum number of data points could be collected over the entire temperature range of the melting curve. To compensate, it was necessary to increase the recommended heating rate of $0.5^{\circ} \mathrm{C} / \mathrm{min}$ to $2.5^{\circ} \mathrm{C} / \mathrm{min}$. Table 3 also 
Table 3. Summary statistics of mole percent purity determination of seven SARMs suited to DSC techniques. The heating rate for each analysis was $2.5^{\circ} \mathrm{C} / \mathrm{min}$.

a. 1,3,5 TNB.

\begin{tabular}{|c|c|c|c|c|c|c|c|c|c|}
\hline \multirow{2}{*}{$\begin{array}{c}\text { Spec. } \\
\text { no. }\end{array}$} & \multirow{2}{*}{$\begin{array}{c}\text { Mass } \\
\text { (mg) }\end{array}$} & \multirow{2}{*}{$\begin{array}{c}\theta_{0} \\
\left({ }^{\circ} \mathrm{C}\right)\end{array}$} & \multirow{2}{*}{$\begin{array}{l}\theta_{0} \\
(K)\end{array}$} & \multicolumn{2}{|c|}{ Fraction $(K)$} & \multirow{2}{*}{$\begin{array}{l}\text { Corr. } \\
(X \%)\end{array}$} & \multirow{2}{*}{$\begin{array}{c}\text { Hf } \\
\text { (cal/mole) }\end{array}$} & \multirow{2}{*}{$\begin{array}{c}H f \\
(\text { callgm })\end{array}$} & \multirow{2}{*}{$\begin{array}{c}\text { Purity } \\
(\%)\end{array}$} \\
\hline & & & & start & end & & & & \\
\hline 1 & 1.55 & 122.941 & 396.101 & 395.948 & 396.197 & 22.3 & 4340.263 & 20.366 & 99.954 \\
\hline 2 & 2.34 & 123.131 & 396.291 & 396.114 & 396.364 & 20.1 & 4284.198 & 20.103 & 99.953 \\
\hline 3 & 2.29 & 123.042 & 396.202 & 396.031 & 396.322 & 35.0 & 4816.054 & 22.599 & 99.925 \\
\hline 4 & 1.65 & 122.958 & 396.118 & 395.962 & 396.253 & 24.6 & 4457.232 & 20.915 & 99.948 \\
\hline 5 & 2.89 & 123.011 & 396.171 & 396.003 & 396.336 & 24.2 & 4515.091 & 21.187 & 99.945 \\
\hline 6 & 1.65 & 123.067 & 396.227 & 396.045 & 396.295 & 24.3 & 4530.101 & 21.257 & 99.938 \\
\hline 7 & 2.45 & 123.131 & 396.291 & 396.128 & 396.503 & 16.1 & 4159.037 & 19.516 & 99.964 \\
\hline 8 & 2.10 & 123.225 & 396.385 & 396.211 & 396.545 & 24.0 & 4335.729 & 20.345 & 99.944 \\
\hline 9 & 1.71 & 123.173 & 396.333 & 396.170 & 396.461 & 30.4 & 4729.276 & 22.192 & 99.935 \\
\hline 10 & 2.38 & 123.136 & 396.296 & 396.128 & 396.461 & 23.1 & 4463.889 & 20.946 & 99.948 \\
\hline & 2.1 & 123.08 & 396.242 & 396.074 & 396.374 & . & 7 & 20.943 & 99.945 \\
\hline Std.Dev. & 0.44 & 0.09 & 0.093 & 0.089 & 0.114 & 5.2 & 199.562 & 0.937 & 0.011 \\
\hline Variance & 0.20 & 0.01 & 0.009 & 0.008 & 0.013 & 26.9 & 39825.108 & 0.877 & 0.000 \\
\hline
\end{tabular}

\section{b. 2,4,6 TNBA.}

\begin{tabular}{|c|c|c|c|c|c|c|c|c|c|}
\hline \multirow{2}{*}{$\begin{array}{c}\text { Spec. } \\
\text { no. }\end{array}$} & \multirow{2}{*}{$\begin{array}{l}\text { Mass } \\
(\mathrm{mg})\end{array}$} & \multirow{2}{*}{$\begin{array}{c}\theta_{0} \\
\left({ }^{\circ} \mathrm{C}\right)\end{array}$} & \multirow{2}{*}{$\begin{array}{l}\theta_{0} \\
\left(K^{\prime}\right)\end{array}$} & \multicolumn{2}{|c|}{ Fraction $(K)$} & \multirow{2}{*}{$\begin{array}{l}\text { Corr. } \\
\text { (X\%) }\end{array}$} & \multirow{2}{*}{$\begin{array}{c}H f \\
\text { (callmole) }\end{array}$} & \multirow{2}{*}{$\begin{array}{c}H f \\
(\mathrm{cal} / \mathrm{gm})\end{array}$} & \multirow{2}{*}{$\begin{array}{c}\text { Purity } \\
(\%)\end{array}$} \\
\hline & & & & start & end & & & & \\
\hline 1 & 2.16 & 118.884 & 392.044 & 391.779 & 392.196 & 12.7 & 4455.758 & 18.479 & 99.891 \\
\hline 2 & 2.42 & 118.713 & 391.873 & 391.654 & 392.487 & 36.3 & 5728.564 & 23.758 & 99.833 \\
\hline 3 & 1.24 & 118.701 & 391.861 & 391.638 & 392.221 & 23.2 & 5755.129 & 23.868 & 99.837 \\
\hline 4 & 1.81 & 119.447 & 392.607 & 392.404 & 393.070 & 19.3 & 5015.647 & 20.801 & 99.882 \\
\hline 5 & 1.75 & 119.158 & 392.318 & 392.071 & 392.404 & 2.3 & 4047.713 & 16.787 & 99.916 \\
\hline 6 & 2.36 & 119.239 & 392.399 & 392.162 & 392.829 & 32.3 & 5102.442 & 21.161 & 99.841 \\
\hline 7 & 2.02 & 118.888 & 392.048 & 391.854 & 392.854 & 44.2 & 5659.786 & 23.473 & 99.840 \\
\hline 8 & 1.46 & 118.589 & 391.749 & 391.461 & 391.794 & 13.7 & 3986.815 & 16.535 & 99.898 \\
\hline 9 & 1.99 & 118.579 & 391.739 & 391.494 & 392.077 & 34.0 & 4549.465 & 18.868 & 99.864 \\
\hline 10 & 2.19 & 119.098 & 392.258 & 391.961 & 392.294 & 19.0 & 4138.200 & 17.162 & 99.865 \\
\hline & 1. & 118.93 & 392.090 & 391.848 & 392.4 & 2. & & 20.089 & 9.867 \\
\hline Std. Dev. & 0.38 & 0.30 & 0.295 & 0.304 & 0.394 & 12.8 & 705.443 & 2.926 & 0.029 \\
\hline Variance & 0.14 & 0.09 & 0.087 & 0.092 & 0.155 & 164.4 & 497649.636 & 8.559 & 0.001 \\
\hline
\end{tabular}

\section{c. 2,4 DNT.}

\begin{tabular}{|c|c|c|c|c|c|c|c|c|c|}
\hline \multirow{2}{*}{$\begin{array}{c}\text { Spec. } \\
\text { no. }\end{array}$} & \multirow{2}{*}{$\begin{array}{l}\text { Mass } \\
(\mathrm{mg})\end{array}$} & \multirow{2}{*}{$\begin{array}{c}\theta_{0} \\
\left({ }^{\circ}\right)\end{array}$} & \multirow{2}{*}{$\begin{array}{l}\theta_{0} \\
(K)\end{array}$} & \multicolumn{2}{|c|}{ Fraction $(K)$} & \multirow{2}{*}{$\begin{array}{l}\text { Corr. } \\
\text { (X\%) }\end{array}$} & \multirow{2}{*}{$\begin{array}{c}\text { Hf } \\
\text { (callmole) }\end{array}$} & \multirow{2}{*}{$\begin{array}{c}H f \\
(\text { callgm })\end{array}$} & \multirow{2}{*}{$\begin{array}{l}\text { Purity } \\
\text { (\%) }\end{array}$} \\
\hline & & & & start & end & & & & \\
\hline 1 & 2.03 & 70.21 & 343.370 & 342.767 & 343.392 & 28.6 & 5285.832 & 29.021 & 99.531 \\
\hline 2 & 2.00 & 69.659 & 342.819 & 342.351 & 343.392 & 30.9 & 5041.063 & 27.677 & 99.686 \\
\hline 3 & 1.46 & 70.331 & 343.491 & 343.184 & 343.600 & 15.8 & 4986.223 & 27.376 & 99.823 \\
\hline 4 & 1.69 & 70.459 & 343.619 & 343.184 & 343.600 & 26.1 & 5182.906 & 28.456 & 99.645 \\
\hline 5 & 1.92 & 70.27 & 343.430 & 342.976 & 343.600 & 20.1 & 4948.470 & 27.168 & 99.765 \\
\hline 6 & 2.20 & 69.422 & 342.582 & 342.143 & 343.808 & 38.1 & 4816.913 & 26.446 & 99.656 \\
\hline 7 & 2.06 & 70.654 & 343.814 & 343.392 & 343.808 & 18.0 & 4865.404 & 26.712 & 99.762 \\
\hline 8 & 2.54 & 70.64 & 343.800 & 343.184 & 343.808 & 21.4 & 4755.163 & 26.107 & 99.647 \\
\hline 9 & 1.95 & 70.184 & 343.344 & 342.767 & 343.808 & 45.1 & 4896.278 & 26.882 & 99.506 \\
\hline 10 & & & & & & & & & \\
\hline & 1.98 & 70.20 & 343.363 & 342.883 & 343.646 & 27.1 & 4975.361 & 27.316 & 99.669 \\
\hline Std. Dev. & 0.30 & 0.42 & 0.416 & 0.418 & 0.173 & 9.7 & 171.960 & 0.944 & 0.105 \\
\hline Variance & 0.09 & 0.17 & 0.173 & 0.175 & 0.030 & 94.3 & 29570.328 & 0.892 & 0.011 \\
\hline
\end{tabular}


Table 3 (cont'd). Summary statistics of mole percent purity determination of seven SARMs suited to DSC techniques. The heating rate for each analysis was $2.5^{\circ} \mathrm{C} / \mathrm{min}$.

\section{d. 2,6 DNT.}

\begin{tabular}{|c|c|c|c|c|c|c|c|c|c|}
\hline \multirow{2}{*}{$\begin{array}{c}\text { Spec. } \\
\text { no. }\end{array}$} & \multirow{2}{*}{$\begin{array}{c}\text { Mass } \\
\text { (mg) }\end{array}$} & \multirow{2}{*}{$\begin{array}{c}\theta_{0} \\
\left({ }^{\circ} \mathrm{C}\right)\end{array}$} & \multirow{2}{*}{$\begin{array}{c}\theta_{o} \\
(K)\end{array}$} & \multicolumn{2}{|c|}{ Fraction $(K)$} & \multirow{2}{*}{$\begin{array}{l}\text { Corr. } \\
\text { (X\%) }\end{array}$} & \multirow{2}{*}{$\begin{array}{c}H f \\
\text { (callmole) }\end{array}$} & \multirow{2}{*}{$\begin{array}{c}H f \\
(\mathrm{cal} / \mathrm{gm})\end{array}$} & \multirow{2}{*}{$\begin{array}{c}\text { Purity } \\
\text { (\%) }\end{array}$} \\
\hline & & & & start & end & & & & \\
\hline 1 & 2.39 & 65.052 & 338.212 & 338.120 & 338.370 & 22.6 & 3689.047 & 20.254 & 99.962 \\
\hline 2 & 2.09 & 65.426 & 338.586 & 338.478 & 338.644 & 14.1 & 1294.827 & 7.109 & 99.992 \\
\hline 3 & 1.31 & 65.265 & 338.425 & 338.299 & 338.466 & 20.6 & 4150.906 & 22.790 & 99.936 \\
\hline \multicolumn{10}{|c|}{ 然 } \\
\hline 5 & 2.45 & 57.923 & 331.083 & 330.823 & 331.239 & 10.7 & 5345.079 & 29.346 & 99.919 \\
\hline 6 & 2.10 & 57.945 & 331.105 & 330.887 & 331.137 & 35.9 & 6528.501 & 35.843 & 99.761 \\
\hline \multicolumn{10}{|l|}{7} \\
\hline 8 & 2.99 & 57.787 & 330.947 & 330.802 & 330.969 & 13.2 & 5418.076 & 29.747 & 99.945 \\
\hline 9 & 2.76 & 57.807 & 330.967 & 330.803 & 331.136 & 21.6 & 5596.084 & 30.724 & 99.914 \\
\hline 10 & 2.16 & 68.969 & 342.129 & 341.926 & 342.759 & 23.4 & 5384.466 & 29.562 & 99.872 \\
\hline Average & 2.28 & 62.02 & 335.182 & 335.017 & 335.340 & 20.3 & 4675.873 & 25.672 & 99.913 \\
\hline Std. Dev. & 0.51 & 4.61 & 4.609 & 4.633 & 4.723 & 7.9 & 1623.960 & 8.916 & 0.071 \\
\hline Variance & 0.26 & 21.24 & 21.239 & 21.469 & 22.310 & 63.0 & 2637244.679 & 79.493 & 0.005 \\
\hline
\end{tabular}

e. Picric acid.

\begin{tabular}{|c|c|c|c|c|c|c|c|c|c|}
\hline \multirow{2}{*}{$\begin{array}{c}\text { Spec. } \\
\text { no. }\end{array}$} & \multirow{2}{*}{$\begin{array}{c}\text { Mass } \\
(\mathrm{mg})\end{array}$} & \multirow{2}{*}{$\begin{array}{c}\theta_{0} \\
\left({ }^{\circ} \mathrm{C}\right)\end{array}$} & \multirow{2}{*}{$\begin{array}{c}\theta_{0} \\
(K)\end{array}$} & \multicolumn{2}{|c|}{ Fraction $(K)$} & \multirow{2}{*}{$\begin{array}{l}\text { Corr. } \\
\text { (X\%) }\end{array}$} & \multirow{2}{*}{$\begin{array}{c}H f \\
\text { (callmole) }\end{array}$} & \multirow{2}{*}{$\begin{array}{c}H f \\
(\mathrm{call} / \mathrm{gm})\end{array}$} & \multirow{2}{*}{$\begin{array}{c}\text { Purity } \\
(\%)\end{array}$} \\
\hline & & & & start & end & & & & \\
\hline 1 & 1.74 & 122.009 & 395.169 & 394.962 & 395.295 & 23.0 & 4649.824 & 20.305 & 99.909 \\
\hline 2 & 3.02 & 122.030 & 395.190 & 394.962 & 395.295 & 28.3 & 4907.968 & 21.432 & 99.881 \\
\hline 3 & 2.04 & 121.647 & 394.807 & 394.626 & 395.042 & 23.7 & 4933.367 & 21.543 & 99.913 \\
\hline 4 & 2.00 & 121.978 & 395.138 & 394.876 & 395.126 & 32.2 & 5324.762 & 23.252 & 99.828 \\
\hline 5 & 1.69 & 122.362 & 395.522 & 395.281 & 395.614 & 30.6 & 5031.882 & 21.973 & 99.863 \\
\hline 6 & 2.37 & 122.103 & 395.263 & 395.034 & 395.367 & 24.1 & 5003.511 & 21.849 & 99.881 \\
\hline 7 & 1.99 & 121.933 & 395.093 & 394.876 & 395.209 & 29.4 & 5039.895 & 22.008 & 99.877 \\
\hline 8 & 1.33 & 121.857 & 395.017 & 394.826 & 395.117 & 28.5 & 5335.077 & 23.297 & 99.887 \\
\hline 9 & 2.37 & 121.503 & 394.663 & 394.459 & 395.042 & 37.4 & 5602.834 & 24.467 & 99.868 \\
\hline 10 & 1.90 & 121.952 & 395.112 & 394.909 & 395.201 & 25.8 & 5118.580 & 22.352 & 99.883 \\
\hline Average & 2.05 & 121.94 & 395.097 & 394.881 & 395.231 & 28.3 & 5094.770 & 22.248 & 99.879 \\
\hline Std. Dev. & 0.46 & 0.24 & 0.236 & 0.222 & 0.173 & 4.4 & 267.407 & 1.168 & 0.024 \\
\hline Variance & 0.21 & 0.06 & 0.056 & 0.049 & 0.030 & 19.7 & 71506.328 & 1.364 & 0.001 \\
\hline
\end{tabular}

\section{f. Tetryl.}

\begin{tabular}{|c|c|c|c|c|c|c|c|c|c|}
\hline \multirow{2}{*}{$\begin{array}{l}\text { Spec. } \\
\text { no. }\end{array}$} & \multirow{2}{*}{$\begin{array}{c}\text { Mass } \\
(\mathrm{mg})\end{array}$} & \multirow{2}{*}{$\begin{array}{l}\theta_{0} \\
\left.{ }^{\circ} \mathrm{C}\right)\end{array}$} & \multirow{2}{*}{$\begin{array}{l}\theta_{0} \\
(K)\end{array}$} & \multicolumn{2}{|c|}{ Fraction $(K)$} & \multirow{2}{*}{$\begin{array}{l}\text { Corr. } \\
\text { (X\%) }\end{array}$} & \multirow{2}{*}{$\begin{array}{c}H f \\
\text { (callmole) }\end{array}$} & \multirow{2}{*}{$\begin{array}{c}\mathrm{Hf} \\
(\mathrm{call} / \mathrm{gm})\end{array}$} & \multirow{2}{*}{$\begin{array}{l}\text { Purity } \\
\text { (\%) }\end{array}$} \\
\hline & & & & start & end & & & & \\
\hline 1 & 2.86 & 129.085 & 402.245 & 402.130 & 402.771 & 9.4 & 6045.691 & 21.054 & 99.975 \\
\hline 2 & 2.34 & 129.102 & 402.262 & 402.130 & 402.563 & 7.6 & 5954.854 & 20.738 & 99.975 \\
\hline 3 & 2.66 & 129.348 & 402.508 & 402.380 & 402.963 & 11.6 & 6226.205 & 21.683 & 99.966 \\
\hline 4 & 3.03 & 129.101 & 402.261 & 402.130 & 402.671 & 9.1 & 6083.879 & 21.187 & 99.970 \\
\hline 5 & 1.91 & 129.189 & 402.349 & 402.213 & 402.630 & 9.9 & 6133.364 & 21.359 & 99.965 \\
\hline 6 & 1.73 & 129.187 & 402.347 & 402.213 & 402.646 & 9.2 & 6079.107 & 21.170 & 99.967 \\
\hline 7 & 2.23 & 129.172 & 402.332 & 402.213 & 402.763 & 9.4 & 6207.048 & 21.616 & 99.973 \\
\hline 8 & 2.42 & 129.525 & 402.685 & 402.546 & 403.021 & 12.5 & 6276.334 & 21.857 & 99.959 \\
\hline 9 & 2.54 & 129.298 & 402.458 & 402.338 & 402.896 & 8.5 & 6068.322 & 21.133 & 99.976 \\
\hline 10 & 1.67 & 129.272 & 402.432 & 402.296 & 402.713 & 9.0 & 6145.558 & 21.402 & 99.967 \\
\hline & 2.34 & 129.23 & . & 402.259 & 402.764 & 9.6 & 36 & 21.320 & 99.969 \\
\hline Std. Dev. & 0.46 & 0.14 & 0.137 & 0.134 & 0.152 & 1.4 & 95.760 & 0.333 & 0.005 \\
\hline Variance & 0.21 & 0.02 & 0.019 & 0.018 & 0.023 & 2.1 & 9170.015 & 0.111 & 0.000 \\
\hline
\end{tabular}


Table 3 (cont'd).

\begin{tabular}{|c|c|c|c|c|c|c|c|c|c|}
\hline \multirow{2}{*}{$\begin{array}{c}\text { Spec. } \\
\text { no. }\end{array}$} & \multirow{2}{*}{$\begin{array}{c}\text { Mass } \\
\text { (mg) }\end{array}$} & \multirow{2}{*}{$\begin{array}{c}\theta_{0} \\
\left({ }^{\circ} \mathrm{C}\right)\end{array}$} & \multirow{2}{*}{$\begin{array}{l}\theta_{0} \\
(K)\end{array}$} & \multicolumn{2}{|c|}{ Fraction (K) } & \multirow{2}{*}{$\begin{array}{l}\text { Corr. } \\
(X \%)\end{array}$} & \multirow{2}{*}{$\begin{array}{c}H f \\
\text { (cal/mole) }\end{array}$} & \multirow{2}{*}{$\begin{array}{c}H f \\
\text { (callgm) }\end{array}$} & \multirow{2}{*}{$\begin{array}{c}\text { Purity } \\
\text { (\%) }\end{array}$} \\
\hline & & & & start & end & & & & \\
\hline 1 & 2.36 & 81.62 & 354.780 & 354.442 & 354.858 & 33.7 & 6795.214 & 29.918 & 99.731 \\
\hline 2 & 2.06 & 81.771 & 354.931 & 354.546 & 355.067 & 36.6 & 6761.890 & 29.771 & 99.686 \\
\hline 3 & 2.04 & 81.593 & 354.753 & 354.442 & 354.858 & 31.8 & 6749.360 & 29.716 & 99.765 \\
\hline 4 & 2.15 & 80.858 & 354.018 & 353.816 & 354.149 & 20.3 & 6279.014 & 27.645 & 99.889 \\
\hline 5 & 2.57 & 80.91 & 354.070 & 353.899 & 354.399 & 27.5 & 6457.268 & 28.430 & 99.893 \\
\hline 6 & 1.43 & 80.8 & 353.960 & 353.816 & 354.149 & 11.2 & 5794.607 & 25.512 & 99.957 \\
\hline 7 & 1.59 & 80.621 & 353.781 & 353.592 & 353.925 & 20.0 & 6469.962 & 28.486 & 99.902 \\
\hline 8 & 2.37 & 80.693 & 353.853 & 353.675 & 354.175 & 24.0 & 6769.176 & 29.803 & 99.892 \\
\hline 9 & 1.71 & 80.563 & 353.723 & 353.509 & 353.842 & 32.2 & 7028.742 & 30.946 & 99.825 \\
\hline 10 & 2.60 & 80.564 & 353.724 & 353.509 & 353.842 & 22.3 & 6595.779 & 29.040 & 99.871 \\
\hline Average & 2.09 & 81.00 & 354.159 & 353.925 & 354.326 & 26.0 & 6570.101 & 28.927 & 99.841 \\
\hline Std.Dev. & 0.41 & 0.47 & 0.473 & 0.403 & 0.452 & 7.8 & 346.268 & 1.525 & 0.087 \\
\hline Variance & 0.16 & 0.22 & 0.224 & 0.163 & 0.204 & 61.2 & 119901.794 & 2.325 & 0.008 \\
\hline
\end{tabular}

shows that the average sample mass was approximately $2 \mathrm{mg}$ instead of the recommended $1.7 \mathrm{mg}$. This was due in part to poor laboratory technique, as well as the granular nature of the compounds, which made it difficult to control the specimen's mass in that small range. According to Hunter and Blaine (1984) the combined effect of this heating rate and mass deviation should cause a $0.20 \mathrm{~mol} \%$ overestimation of the purity. The values listed in Table 3 accordingly must be adjusted downward and the number of significant figures reduced to two. Table 4 presents the results of these adjustments to the values in Table 3 and shows that each SARM's calculated purity is still well within the $98 \mathrm{~mol} \%$ criterion of the USATHAMA QA manual.

Table 4. Final purity values of SARMs.

\begin{tabular}{lc} 
SARM & Purity $(\mathrm{mol} \%)$ \\
\hline 1,3,5 TNB & 99.74 \\
2,4,6 TNBA & 99.47 \\
2,4 DNT & 99.67 \\
2,6 DNT & 99.71 \\
Picric acid & 99.68 \\
Tetryl & 99.77 \\
TNT & 99.64 \\
\hline
\end{tabular}

\section{CONCLUSION}

It is interesting to note the effect that compounds such as the SARMs, with large values of melting temperature and heat of fusion, have on eq 1. The most apparent is that temperature inaccuracies must be significant $(>2 \mathrm{~K})$ to affect the predicted purity. Likewise, but to a lesser degree, there must be a large uncertainty in the calcu- lated heat of fusion to modify the predicted purity value. These artifacts might indicate that the DSC approach is inherently well suited for determining the purity of the SARMs.

The procedure used to analyze the SARMs (Pidgeon and Black, in prep.) in this experiment was found to be accurate as seen by a comparison of calculated values with the values listed in Table 1 from other sources. The melting temperatures determined in these tests were all well within the range of literature values. The same is true of the calculated heats of fusion, but they were not as close to literature values.

Differential scanning calorimetry is a suitable technique for determining mole percent purity of the seven Standard Analytical Reference Materials that meet the three constraints imposed by eq 1 . In addition, the SARMs tested were found to exceed the minimum purity value set by USATHAMA and therefore are suitable standards for their program.

\section{LITERATURE CITED}

Gibbs, T.R. and A.Popolato (Ed.) (1980) LASL Explosive Property Data. Berkeley: University of California Press.

Hunter, J. and R. Blaine (1984) Optimization of accuracy and precision in the differential scanning calorimetry dynamic purity method. In Purity Determination by ThermalMethods (R.L. Blaine and C.K. Schoff, Ed.). ASTM Special Technical Publication 838, p. 29-38. Jenkins, T.F., P.W. Schumacher, M.E. Walsh and C.F. Bauer (1988) Development of an analytical method for the determination of explosive residues in soil. Part II: Additional development and ruggedness testing. USA Cold Regions Research and Engineering Laboratory, CRREL Report 88-8. 
Meyer, R. (1987) Explosives, 3rd Edition. New York: Weinheim Press.

Pidgeon, D. and P.B. Black (in prep.) Operators manual for determining mole percent purity using IMPURE. USA Cold Regions Research and Engineering Laboratory, CRREL Special Report.

Pidgeon, D. and P.B. Black (1989) Initial mole percent purity measurements of the Standard Analytical Reference Materials. USA Cold Regions Research and Engineering Laboratory, CRREL Internal Report 1039 (unpublished).

Plato, C. and A.R. Glasgow (1969) Differential scanning calorimetry as a general method for determining the purity and heat of fusion of high-purity organic chemicals. Application to 95 compounds. Analytical Chemistry, 41: $330-336$.
Perkin-Elmer Corporation (n.d.) Determination of purity by differential scanning calorimetry. Thermal Analysis Newsletter, no. 5. Norwalk, Connecticut.

USATHAMA (1987) QA Program, 2nd Edition. USA Toxic and Hazardous Materials Agency, Aberdeen Proving Ground, Maryland.

Wentsel, R.S., R.G. Hyde, W.E. Jone, III, M.J. Wilkinson, W.E. Harward, III, J.F. Kitchens (1979) Problem definition study on 1,3-dinitrobenzene, 1,3,5trinitrobenzene and di-n-propyl adate. EPA contract no. DAMD17-77-C-7057.

Widmann, G. and H. Sommerauer (1988) Application of DSC purity analysis. American Laboratory, May, p. 106-112. 


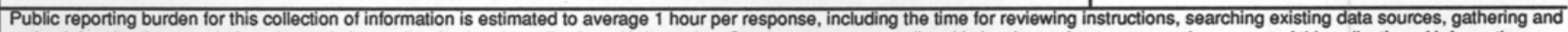
maintaining the data needed, and completing and reviewing the collection of information. Send comments regarding this burden estimate or any other aspect of this collection of information,

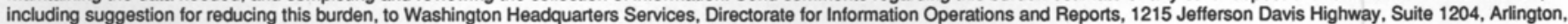



\begin{tabular}{|l|l|l}
\hline 1. AGENCY USE ONLY (Leave blank) & $\begin{array}{c}\text { 2. REPORT DATE } \\
\text { May } 1990\end{array}$ & 3. REPORT TYPE AND DATES COVERED \\
\hline
\end{tabular}

4. TITLE AND SUBTITLE

Purity Determination of Standard Analytical Reference Materials by

Differential Scanning Calorimetry

6. AUTHORS

Patrick B. Black and Dean Pidgeon

7. PERFORMING ORGANIZATION NAME(S) AND ADDRESS(ES)

U.S. Army Cold Regions Research and Engineering Laboratory

72 Lyme Road

Hanover, New Hampshire 03755-1290

8. PERFORMING ORGANIZATION REPORT NUMBER

Special Report 90-16

9. SPONSORING/MONITORING AGENCY NAME(S) AND ADDRESS(ES)

United States Army Toxic and Hazardous Materials Agency

Aberdeen Proving Ground, Maryland

5. FUNDING NUMBERS

Special Report $90-16$

10. SPONSORING/MONITORING AGENCY REPORT NUMBER

CETHA-TE-CR89044

11. SUPPLEMENTARY NOTES

12a. DISTRIBUTION/AVAILABILITY STATEMENT 12b. DISTRIBUTION CODE

Approved for public release; distribution is unlimited.

Available from NTIS, Springfield, Virginia 22161

13. ABSTRACT (Maximum 200 words)

As part of the United States Army Toxic and Hazardous Materials Agency (USATHAMA) Quality Assurance program to maintain a set of high-purity ( $>98 \mathrm{~mol} \%$ ) Standard Analytical Reference Materials (SARMs), the SARMs' purity must be routinely monitored. This report presents data on melting temperature, freezing point depression and heat of fusion as measured by differential scanning calorimetry (DSC) for the seven SARMs suited to DSC methods. These data were then used in the van't Hoff's equation to determine each munition standard's molar purity. The purity of each tested SARM was greater that $98 \mathrm{~mol} \%$, which confirmed the integrity of the SARMs.

\section{SUBJECT TERMS}

Differential scanning calorimetry Munitions production
17. SECURITY CLASSIFICATION OF REPORT

\section{UNCLASSIFIED}

18. SECURITY CLASSIFICATION OF THIS PAGE

UNCLASSIFIED
Standard Analytical Reference Materials

19. SECURITY CLASSIFICATION

OF ABSTRACT

UNCLASSIFIED
15. NUMBER OF PAGES 11

16. PRICE CODE

20. LIMITATION OF ABSTRACT

UL 